\title{
Laboratory colonization by Dirofilaria immitis alters the microbiome of female Aedes aegypti mosquitoes
}

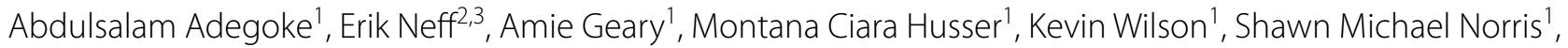
Guha Dharmarajan² and Shahid Karim ${ }^{*^{*}}$ (1)

\begin{abstract}
Background: The ability of blood-feeding arthropods to successfully acquire and transmit pathogens of medical and veterinary importance has been shown to be interfered with, or enhanced by, the arthropod's native microbiome. Mosquitoes transmit viruses, protozoan and filarial nematodes, the majority of which contribute to the $17 \%$ of infectious disease cases worldwide. Dirofilaria immitis, a mosquito-transmitted filarial nematodes of dogs and cats, is vectored by several mosquito species including Aedes aegypti.
\end{abstract}

Methods: In this study, we investigated the impact of D. immitis colonization on the microbiome of laboratory reared female Ae. aegypti. Metagenomic analysis of the V3-V4 variable region of the microbial 165 RNA gene was used for identification of the microbial differences down to species level.

Results: We generated a total of 1068 OTUs representing 16 phyla, 181 genera and 271 bacterial species. Overall, in order of abundance, Proteobacteria, Bacteroidetes, Actinobacteria and Firmicutes were the most represented phylum with D. immitis-infected mosquitoes having more of Proteobacteria (71\%) than uninfected mosquitoes (56.9\%). An interesting finding in this study is the detection of Klebsiella oxytoca in relatively similar abundance in infected and uninfected mosquitoes, suggesting a possible endosymbiotic relationship, and has been previously shown to indirectly compete for nutrients with fungi on domestic housefly eggs and larvae. While D. immitis colonization has no effect on the overall species richness, we identified significant differences in the composition of selected bacterial genera and phyla between the two groups. We also reported distinct compositional and phylogenetic differences in the individual bacterial species when commonly identified bacteria were compared.

Conclusions: To the best of our knowledge, this is the first study to understand the impact of a filarial infection on the microbiome of its mosquito vector. Further studies are required to identify bacteria species that could play an important role in the mosquito biology. While the microbiome composition of Ae. aegypti mosquito have been previously reported, our study shows that in an effort to establish itself, a filarial nematode modifies and alters the overall microbial diversity within its mosquito host.

Keywords: Mosquitoes, Aedes aegypti, Microbiome, Metagenome, Dirofilaria immitis, Dog heartworm

\footnotetext{
*Correspondence: Shahid.Karim@usm.edu

${ }^{1}$ Center for Molecular and Cellular Biosciences, School of Biological,

Environmental, and Earth Sciences, The University of Southern Mississippi, Hattiesburg, MS 39406, USA

Full list of author information is available at the end of the article
}

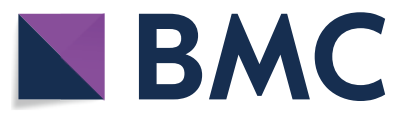

(c) The Author(s) 2020. This article is licensed under a Creative Commons Attribution 4.0 International License, which permits use, sharing, adaptation, distribution and reproduction in any medium or format, as long as you give appropriate credit to the original author(s) and the source, provide a link to the Creative Commons licence, and indicate if changes were made. The images or other third party material in this article are included in the article's Creative Commons licence, unless indicated otherwise in a credit line to the material. If material is not included in the article's Creative Commons licence and your intended use is not permitted by statutory regulation or exceeds the permitted use, you will need to obtain permission directly from the copyright holder. To view a copy of this licence, visit http://creativeco mmons.org/licenses/by/4.0/. The Creative Commons Public Domain Dedication waiver (http://creativecommons.org/publicdomain/ zero/1.0/) applies to the data made available in this article, unless otherwise stated in a credit line to the data. 


\section{Background}

Dirofilaria immitis, responsible for canine respiratory and associated heartworm disease, is a filarial nematode that mainly infects wild and domestic canids, such as dogs and coyotes [1]. It causes serious life-threatening disease of canines and other domestic pets in which adult worms lodge in the cardiopulmonary circulation causing cardiac necrosis which leads to ischemic hypoxia and a total shutdown of the respiratory and cardiovascular system [2]. While primarily a parasite of veterinary importance, human infection has also been reported, albeit rarely [3]. With over 100,000 dogs affected annually, D. immitis has been identified as the most important parasite affecting dogs in the USA [4]. Adult female D. immitis parasites release microfilariae $(\mathrm{mf})$ into the blood stream of the vertebrate host, usually a dog, which are subsequently taken up by adult female mosquitoes during their blood meal $[1,5]$. Microfilariae in the blood enter the midgut of the mosquito during feeding and subsequently migrate to the Malpighian tubules. In the Malpighian tubules of the mosquito mf develop into first-stage larvae and molt twice to reach their infective third-stage (L3), which migrate to the head and proboscis of the mosquito and can infect the next dog during the next blood meal $[1,5]$. The time it takes for the parasite to develop, known as the extrinsic incubation period (EIP), averages 14 days within this mosquito [6].

Beyond the USA, canine dirofilariasis also poses significant problems in dogs in Australia, Europe and Asia [7]. The epidemiology and maintenance of $D$. immitis have been made significant by the abundance of the mosquito vectors with over 70 species of mosquitoes, having been reported to transmit $D$. immitis [8]; of these, 28 are found in the USA [9]. The southeastern USA have been shown to have an increased incidence of canine heartworm disease when compared to other states [10], as about half of the mosquito species known to transmit $D$. immitis are found in the southeastern USA [11], one of which is the mosquito Aedes aegypti [12].

In addition to being an important vector of filarial nematodes such as D. immitis, mosquitoes and other arthropod vectors also host highly complex and diverse microbial communities. Members of these microbial communities have obligate relationships with mosquitoes and are maintained vertically through mosquito generations [13] or acquired from the environment [14, 15]. The microbiome of mosquitoes has been shown to help maintain normal midgut functions, as well as interfering or aiding vector competence of the mosquito [16, 17]. As previously shown, pathogen susceptibility and vector competence of an arthropod can be modulated by the microbial composition within the arthropod vector [18]. Aedes aegypti is the competent vector of many pathogens of humans (Zika, dengue, chikungunya viruses [19-21] and animals (e.g. D. immitis; [22]).

Previous studies have demonstrated significant success in reducing vector competence of mosquitoes for pathogenic microbes by taking advantage of the mosquito microbiome and pathogen interactions [23, 24]. It is a fact that pathogens must compete with the native microbial community in their vector host to propagate and facilitate subsequent transmission to a susceptible host $[18,25]$. Hence, understanding the pathogen-microbiome interaction in vectors of significant public health importance is important.

The prevention and control of canine heartworm disease have primarily focused on the use of therapeutics such as ivermectin and other macrocyclic lactones. While these therapeutics have proven to be effective in killing and preventing the establishment of the third- and early fourth-stage larva [26], recent studies have shown significant resistance against these drugs of choice [27, 28]. Understanding the role(s) played by the mosquito microbiome in facilitating or interfering with $D$. immitis colonization and subsequent transmission will add to existing knowledge on the control of mosquito-transmitted filarial nematodes.

The present study compared the microbiome changes in the mosquito Ae. aegypti, infected with $D$. immitis under laboratory conditions. This study provides an exciting opportunity to advance our knowledge of the major changes in the microbiome of Ae. aegypti following the acquisition of $D$. immitis. This research seeks to address the question "Does $D$. immitis infection of Ae. aegypti mosquito alters its overall microbial richness and abundance?"

\section{Methods \\ Materials}

Unless stated otherwise, equipment and chemicals used for this study were either products of Thermo Fisher Scientific (Grand Island, NY, USA) or Bio-Rad (Hercules, CA, USA).

\section{Mosquito rearing and maintenance}

The mosquito used for investigation was the Ae. aegypti Liverpool Blackeye strain, a highly susceptible mosquito strain to $D$. immitis used predominately in research for Aedes spp. [29]. Although Ae. aegypti is just one of 28 mosquito species reported to vector $D$. immitis in the USA, the relative expertise on this particular species limited us to its use as our study organism. Aedes aegypti, originally obtained from the Filariasis Research Reagent Resource Center (FR3) [30], were raised under standard laboratory conditions: temperature of $27^{\circ} \mathrm{C}$, relative humidity of $80 \pm 5 \%$, and a 12:12-hour light:dark diurnal cycle [31]. 


\section{Mosquito infection with $D$. immitis}

Adult female mosquitoes, five days post-emergence were blood-fed using an artificial membrane feeder. One day prior to membrane-feeding 31 female mosquitoes were transferred to $\sim 500 \mathrm{ml}$ plastic containers with mesh tops (henceforth "cages"). Females were starved of sugar for $12 \mathrm{~h}$ and deprived of water for $4 \mathrm{~h}$ prior to blood-feeding. Mosquitoes in each cage (31 each) were allowed to feed for $2 \mathrm{~h}$ or until repletion on a Parafilm membrane stretched over an inverted water-jacketed glass membrane feeder maintained at $40{ }^{\circ} \mathrm{C}$. Each feeder was filled with $200 \mu \mathrm{l}$ of dog blood infected or uninfected with $D$. immitis. The level of parasitaemia in the infected blood was estimated to be $4500 \mathrm{mf} / \mathrm{ml}$ as previously reported [31]. The dog blood was obtained from FR3.

\section{PCR-based confirmation of Dirofilaria immitis detection}

Prior to screening for $D$. immitis infection from mosquitoes, DNA from individual mosquito samples was extracted using a DNeasy Blood \& Tissue Kit (Qiagen, Germantown, Maryland, USA) and quality was confirmed using a nanodrop machine (Nanodrop One, Thermo Fisher Scientific). All blood-fed (infected and uninfected blood) mosquitoes were screened for $D$. immitis infection irrespective of whether they were fed on infected or uninfected blood by amplifying the cox 1 gene (656 bp) of the D. immitis mitochondrial DNA [32]. Briefly, a $25 \mu \mathrm{l}$ reaction was set up comprising $1 \mu \mathrm{l}$ each of the COI-Forward (5'-TGA TTG GTG GTT TTG GTA A- $3^{\prime}$ ) and COI-Reverse (5'-ATA AGT ACG AGT ATC AAT ATC- $3^{\prime}$ ) primers, $12.5 \mu \mathrm{l}$ of $2 \times$ mastermix (New England Biolabs, Ipswich, Massachusetts, USA), $2.5 \mu \mathrm{l}$ DNA template and $8.5 \mu \mathrm{l}$ of nuclease-free water. For each cycle that was run, a $D$. immitis infected blood sample and nuclease-free water were simultaneously included as positive and negative controls respectively. The PCR cycle comprised of an initial denaturation step at $94{ }^{\circ} \mathrm{C}$ for $5 \mathrm{~min}$ and 40 cycles of $94{ }^{\circ} \mathrm{C}$ for $1 \mathrm{~min}$, annealing at $50{ }^{\circ} \mathrm{C}$ for $2 \mathrm{~min}$ and extension at $72{ }^{\circ} \mathrm{C}$ for $3 \mathrm{~min}$, followed by a final extension step at $72{ }^{\circ} \mathrm{C}$ for $5 \mathrm{~min}$ and an infinite hold at $4{ }^{\circ} \mathrm{C}$.

Confirmation of amplification was done by loading the PCR products in a SYBR safe stained gel. Briefly, $2 \%$ gel was made by autoclaving a solution of $1 \times$ TAE buffer and molecular grade agar. SYBR safe stain $(1 \mu$ SYBR safe: 10 $\mathrm{ml}$ TAE buffer) was added to the agar solution, poured into a precast gel tray and allowed to cool. To load the samples onto the gel, $6 \mu \mathrm{l}$ of PCR product was mixed with $4 \mu \mathrm{l}$ of $6 \times$ dye and pipetted into the wells. Lastly, $5 \mu \mathrm{l}$ of a low molecular weight DNA ladder was loaded onto the gel and the gel could run for $45 \mathrm{~min}$ at $100 \mathrm{~V}$. Amplified PCR products were viewed using a Chemidoc gel imager (Additional file 1: Figure S1).

\section{5 rRNA library preparation and sequencing}

Six individual mosquito genomic DNA extracts were pooled to make one biological replicate and five biological replicates each of $D$. immitis infected and uninfected pools were prepared for metagenomic analysis. The hypervariable V1-V3 region of the $16 S$ rRNA gene was PCR amplified using the forward primer 27F (5'AGR GTT TGA TCM TGG CTC AG-3') and the reverse primer 519R (5'-GTN TTA CNG CGG CKG CTG-3') as outlined by the $16 S$ Illumina's MiSeq protocol (https:// www.mrdnalab.com, Shallowater, TX, USA). Briefly, PCR was performed using the HotStarTaq Plus Master Mix Kit (Qiagen) under the following conditions: $94{ }^{\circ} \mathrm{C}$ for 3 min, followed by $30-35$ cycles of $94{ }^{\circ} \mathrm{C}$ for $30 \mathrm{~s}, 53{ }^{\circ} \mathrm{C}$ for $40 \mathrm{~s}$ and $72{ }^{\circ} \mathrm{C}$ for $1 \mathrm{~min}$, after which a final elongation step at $72{ }^{\circ} \mathrm{C}$ for 5 min was performed. After amplification, PCR products were electrophoresed in $2 \%$ agarose gel to determine the success of amplification and the relative intensity of bands. Multiple samples were pooled together in equal proportions based on their molecular weight and DNA concentrations. Pooled samples were purified using calibrated Ampure XP beads. Then the pooled and purified PCR product was used to prepare Illumina DNA library. Sequencing was performed at MR DNA (https://www.mrdnalab.com, Shallowater, TX, USA) on a MiSeq following the manufacturer's guidelines.

\section{Sequence analysis}

Sequence analysis was carried out using the Quantitative Insights into Microbial Ecology (QIIME 2) pipeline, unless stated otherwise. Briefly, processing of raw fastq files were demultiplexed. The Atacama soil microbiome pipeline was incorporated for quality control of demultiplexed paired-end reads (Additional file 1: Figure S2) using the DADA2 plugin as previously described [33].

Sequence alignment and subsequent construction of phylogenetic tree from representative sequences was performed using the MAFFT v7 and FasTree v2.1 plugin [34] Operational taxonomic assignment was performed using the qiime2 feature-classifier plugin v7.0 which was previously trained against the SILVA 132 database preclustered at 99\%. Tables representing operational taxonomic units (OTUs) and representative taxonomy were exported from $\mathrm{R}$ and used for diversity metric analysis using the Microbiome Analyst web-based interface $[35,36]$. Raw data from this analysis were deposited and assigned the GenBank BioProject number \#PRJNA606536.

\section{Alpha diversity}

To establish whether alpha diversity differs across mosquito samples, reads were transformed and low 
abundance OTUs were filtered from the datasets. The Observed OTU metric was used to estimate species richness by identifying unique OTUs present across the mosquito groups, while the Shannon index was used to estimate both richness and evenness.

\section{Beta diversity}

To compare the differences in the microbiome between mosquito groups based on measures of distance or dissimilarity, dissimilarity matrix was generated from logtransformed sequence data and ordination of the plots were visualized using both the principal coordinates analysis (PCoA) and the non-metric multidimensional scaling (NMDS). The matrix used in calculating beta diversity includes the Bray-Curtis and unweighted UniFrac distance matrix.

\section{Statistical analysis}

To test if species richness and diversity was significant, the Mann-Whitney or Kruskal-Wallis tests was applied to both alpha diversity and classical univariate statistical comparisons analysis, while the significance of beta diversity analysis was determined using the permutational MANOVA (PERMANOVA) test $[35,36]$.

\section{Results}

\section{Dirofilaria immitis effectively colonizes Aedes aegypti mosquitoes under laboratory conditions}

A total of 31 mosquitoes were artificially infected to generate $D$. immitis-infected mosquito. PCR analysis and confirmation of infection status using gel electrophoresis revealed an infection prevalence of $87 \%$ (Additional file 1 : Figure S1).

\section{Microbiome composition}

Analysis of the demultiplexed paired-end-reads generated a total of 602,502 reads which ranged from 31,861 to 110,235 , with an average of 54,758 reads per mosquito. Mosquitoes infected with $D$. immitis had the highest number of reads $(382,714)$ compared to uninfected mosquitoes with a total of 219,788 reads. Taxonomic classification using the SILVA 132 reference database (99\% OTUs full-length sequences) identified 268 operational taxonomic units (OTUs), 11 phyla, 16 genera, and 136 species (Additional file 1: Figure S3).

\section{Dirofilaria immitis infection alters relative abundance of bacteria taxa in Aedes aegypti}

Taxonomic assignment was performed against the SILVA database to observe for the difference in microbiome composition and relative abundance of bacteria species. Both infected and uninfected mosquitoes possessed similar composition of bacteria taxa with differences observed in the relative abundances of specific bacteria species.

The phylum Proteobacteria and Bacteroidetes were present in higher abundance in both mosquito groups. Mosquitoes infected with $D$. immitis had relatively higher abundance of Proteobacteria (71\%) with lower amount of Bacteroidetes (27\%), while uninfected mosquitoes had lower amount of Proteobacteria (56.9\%) and a higher abundance of Bacteroidetes (37\%) (Fig. 1). Among the detected bacteria genera, the genus Klebsiella were detected at relatively similar abundances in both infected (36.3\%) and uninfected (34.6\%) mosquitoes (Fig. 2a).

The genera Ochrobactrum, Sphingobacterium and Pseudomonas were present exclusively in uninfected mosquitoes albeit at an abundance of 4.4\%, 3.6\% and 3\%, respectively (Fig. 2). The genera Enterobacter $(26.1 \%)$ and Elizabethkingia (9.4\%) were in higher abundance in D. immitis-infected mosquitoes (Fig. 2a). Enterobacter hormaechei (24.4\%) and Elizabethkingia meningoseptica $(9.4 \%)$ were more abundant in infected mosquitoes, while Chryseobacterium indologenes (27.4\%) and Grimontella senegalensis (3.9\%), were more abundant in uninfected mosquitoes (Fig. 2b). Additional information on the relative bacteria distribution in individual mosquito can be found in Additional file 1: Figures S4-S7.

\section{Dirofilaria immitis infection affects species diversity in Aedes aegypti}

Following demultiplexing of paired-end reads and quality control by removing chimeric sequences, we normalized OTU counts for individual biological replicates and a rarefaction curve was generated at a depth of 20,000 (Fig. 3). Adequate depth coverage was reached as evidenced by the individual curves plateauing out on the rarefaction curve.

Our results indicated that phylogenetic diversity, estimated using the Shannon index and number of observed OTUs, was reduced in infected mosquitoes compared against uninfected mosquitoes (Fig. 4a, b). Surprisingly, both metrics of alpha diversity used showed no significance when the Kruskal-Wallis test was applied (Observed OTUs: Mann-Whitney $U=3, P=0.053$; Shannon index: Mann-Whitney $U=7, P=0.309$ ).

We also visualized the similarities and differences in the microbial composition of infected and uninfected mosquitoes by carrying out principal coordinates analysis (PCoA) of the unweighted UniFrac and Bray-Curtis distance matrices (Fig. 5c, d). Figure 5c, d shows distinct clustering of $D$. immitis-infected mosquito replicates with no outliers. Beta diversity was significantly changed in D. immitis-infected mosquitoes compared 


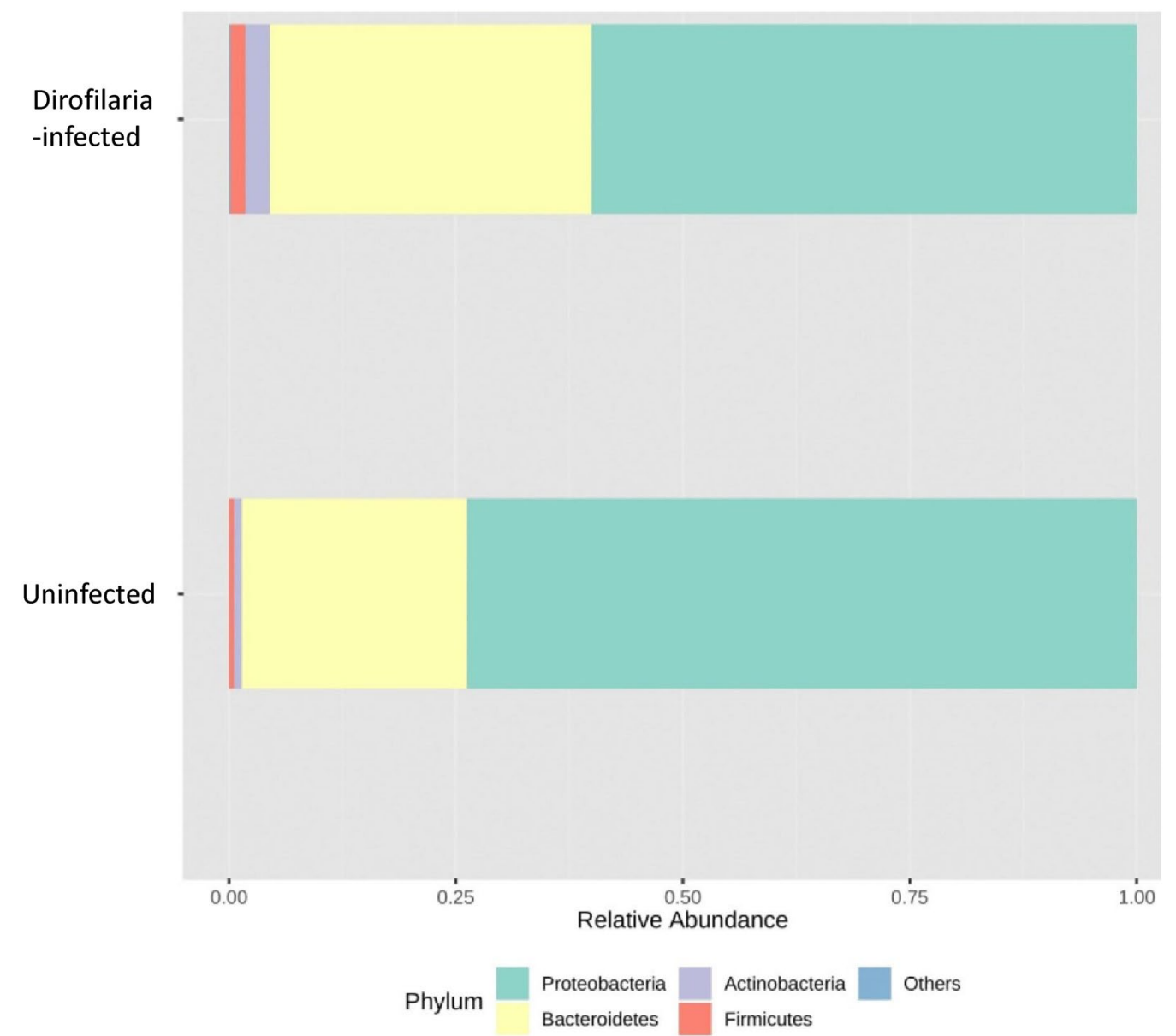

Fig. 1 Phylum level distribution. Relative abundance of bacteria at the phylum level as identified in Dirofilaria immitis-infected and uninfected Aedes aegypti mosquitoes. The phyla Proteobacteria and Bacteroidetes were the most abundant in both groups. Phyla with less than $1 \%$ abundance were grouped as "Others"

to uninfected mosquitoes using the Unweighted UniFrac distance matrix (PERMANOVA, pseudo-FDR $\left.1.4043, R^{2}=0.14932, P=0.286\right)$.

\section{Community profiling and correlation analysis}

To assess the extent to which highly abundant bacteria phyla and genera were represented in Ae. aegypti, we used a combination of pattern correlation and heat map analysis. A very strong positive correlation was seen between the genera Ralstonia, Francisella, Pantoea, Elizabethkingia, Wolbachia, Herbaspirillum and Achromobacter and D. immitis-infected mosquitoes (Fig. 6a). Heat map analysis and phylogenetic tree of the highly represented and dominant bacterial genera also showed the above identified genera to be well represented in more than one of the D. immitis-infected mosquitoes (Additional file 1: Figures S8 and S9).

To explore how top taxa differed between both infected and uninfected mosquitoes, classical univariate statistical comparisons analysis was applied to identify phyla and genera that exhibit significant differences (Mann-Whitney test) in their composition. The phyla Actinobacteria $(\mathrm{FDR}=0.1440, \quad P=0.036)$ and Firmicutes (FDR $=0.14401, P=0.005)$ had significantly higher abundance in the uninfected mosquitoes (Fig. 6b, c). Our analysis also showed that the D. immitis-infected mosquitoes had significantly higher abundance of the genera Elizabethkingia (FDR $=0.175, P=0.015)$ and Wolbachia $(\mathrm{FDR}=0.11448, P=0.011)$, while Pseudomonas $(\mathrm{FDR}=0.12698, P=0.015)$ was much abundant in the uninfected mosquito (Fig. 7a-c).

\section{Discussion}

To the best of our knowledge, this study is the first to utilize $16 \mathrm{~S}$ metagenomic analysis to understand the significance of a filarial nematode on the microbiome of a mosquito vector under laboratory conditions. This 


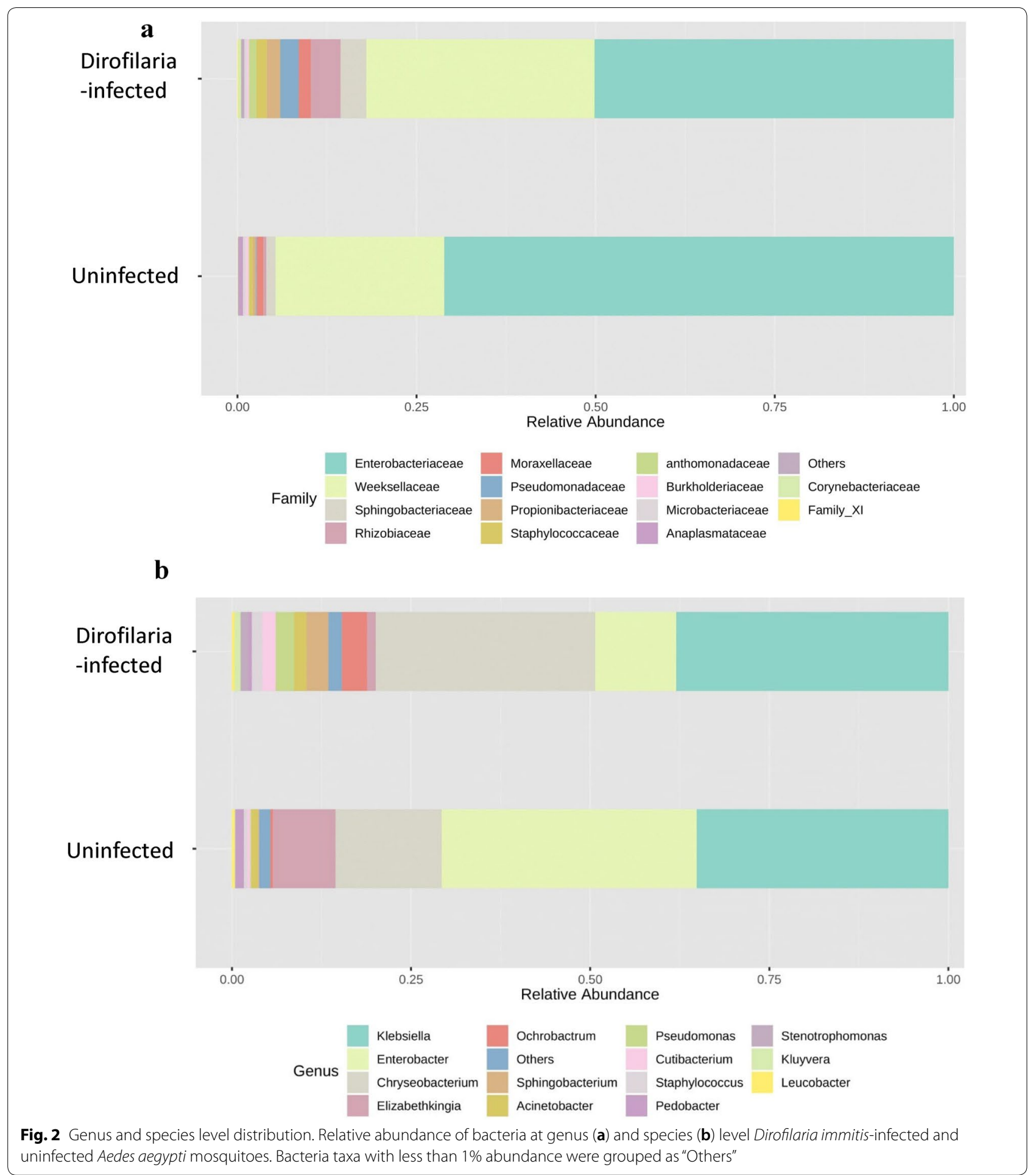

technique offers the advantage of detecting both culturable and unculturable pathogenic and non-pathogenic microbes from a given DNA or RNA sample. As previously reported from previous metagenome studies on mosquitoes and similar arthropods or insects [37-39],
Fig. 1a shows that the phyla Proteobacteria, Bacteroidetes, Actinobacteria and Firmicutes were detected from the mosquito groups tested. Similar studies have also identified the phylum Proteobacteria as one of the dominant phyla in the microbiome of Ae. aegypti mosquitoes 


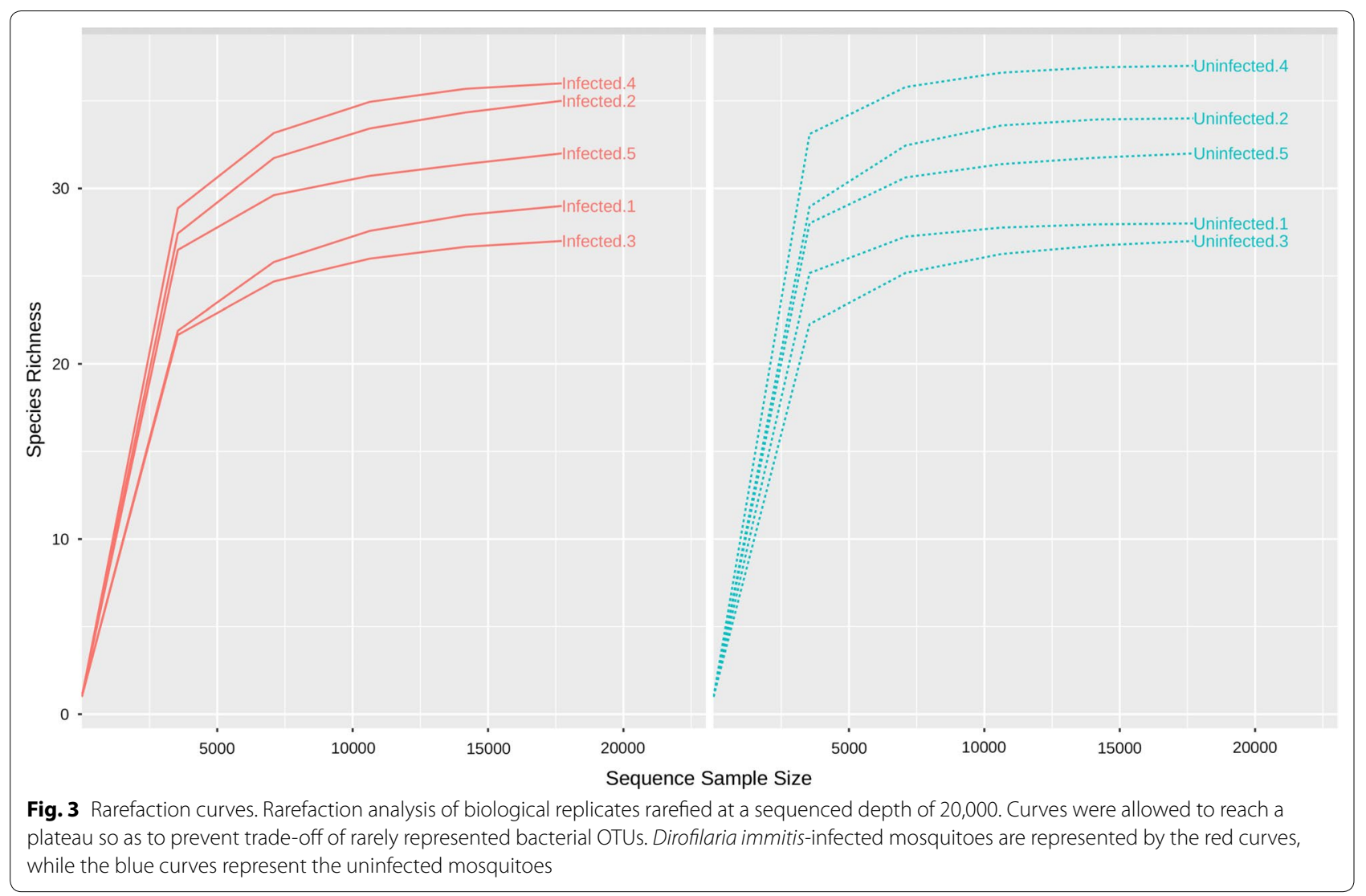

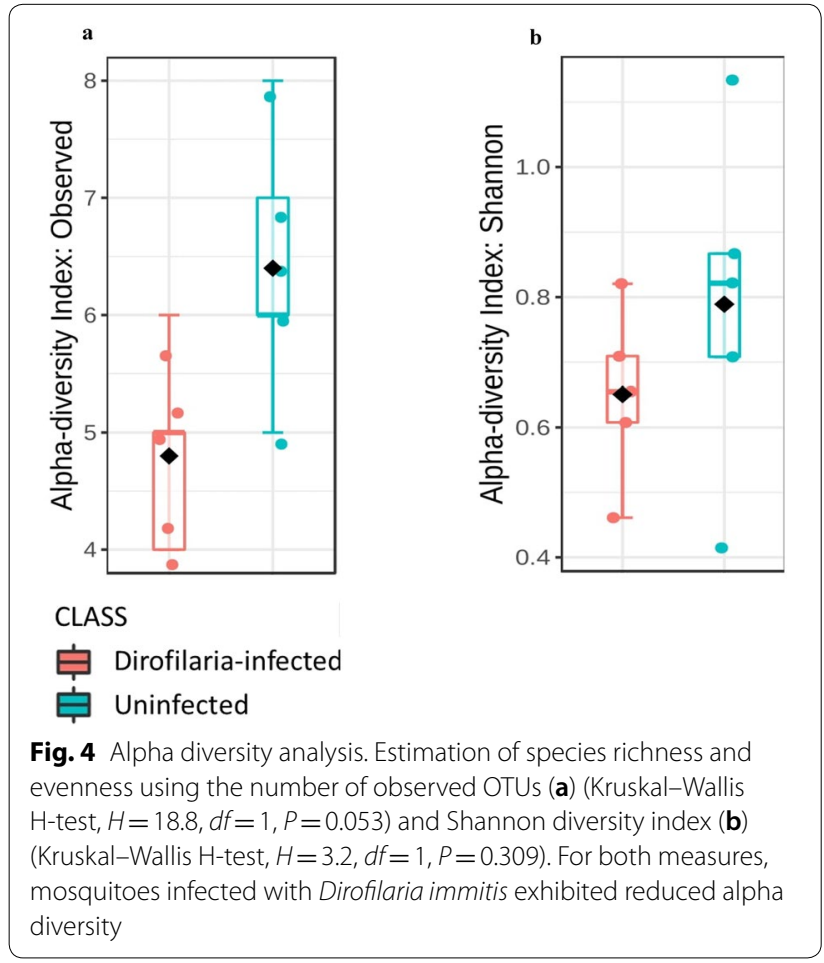

$[23,40]$. We observed an inverse relationship in the abundance of the phyla Proteobacteria and Bacteroidetes in our study with $D$. immitis-infected mosquitoes having a higher abundance of the phylum Proteobacteria when compared to the uninfected mosquitoes and vice versa (Additional file 1: Figure S4).

The Proteobacteria group are the largest phylum found in different environments, plants and animals [41] with members ranging from free-living commensals to pathogenic microbes. We found that the genus Klebsiella was present in relatively similar abundances in both infected and uninfected mosquitoes. Although no known function has been associated to this group in insects or arthropods, Klebsiella belongs to a class of gram-negative Proteobacteria (Class Gammaproteobacteria) with previous detection in field- and laboratory-raised Culex quinquefasciatus, Ae. albopictus and Ae. aegypti [42-44].

We also identified the genus Enterobacter in D. immitis-infected mosquitoes at a relative abundance of 20-fold compared with the uninfected mosquitoes (Fig. 2). This genus has been detected in similar microbiome studies of Ae. aegypti where they have been associated with their role in blood-meal digestion due to their hemolytic activities [45] which could explain why the genus Enterobacter has been commonly associated with females 

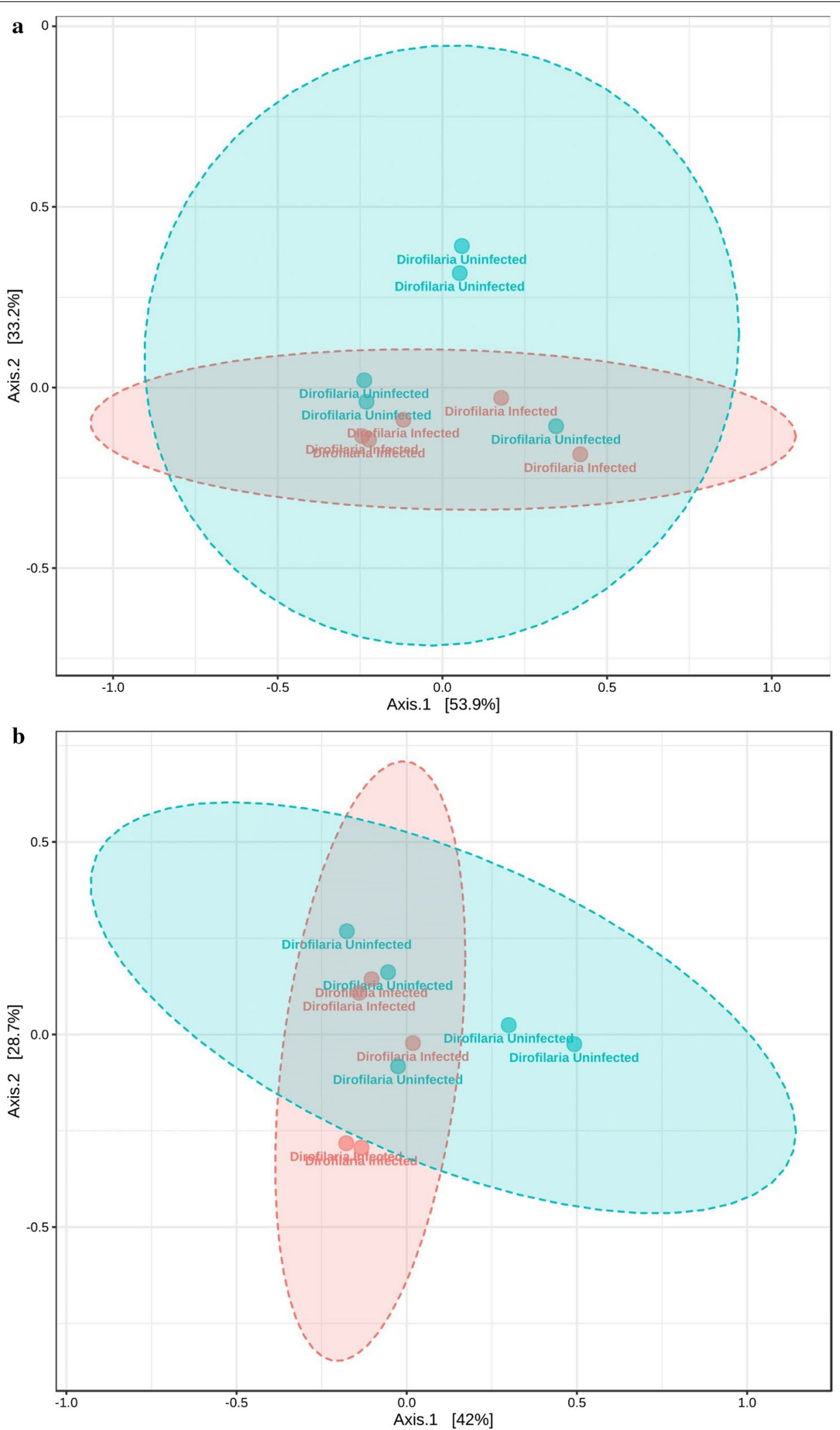

Fig. 5 Beta diversity analysis. Estimation of differences in the microbial communities between infected and uninfected mosquitoes using principal coordinates analysis (PCOA) of the unweighted UniFrac (a) (PERMANOVA, pseudo- $F D R=1.4043, d f=1, R=0.14932, P=0.286$ ) and Bray-Curtis (b) $(P<0.05)$ distance matrices 
a

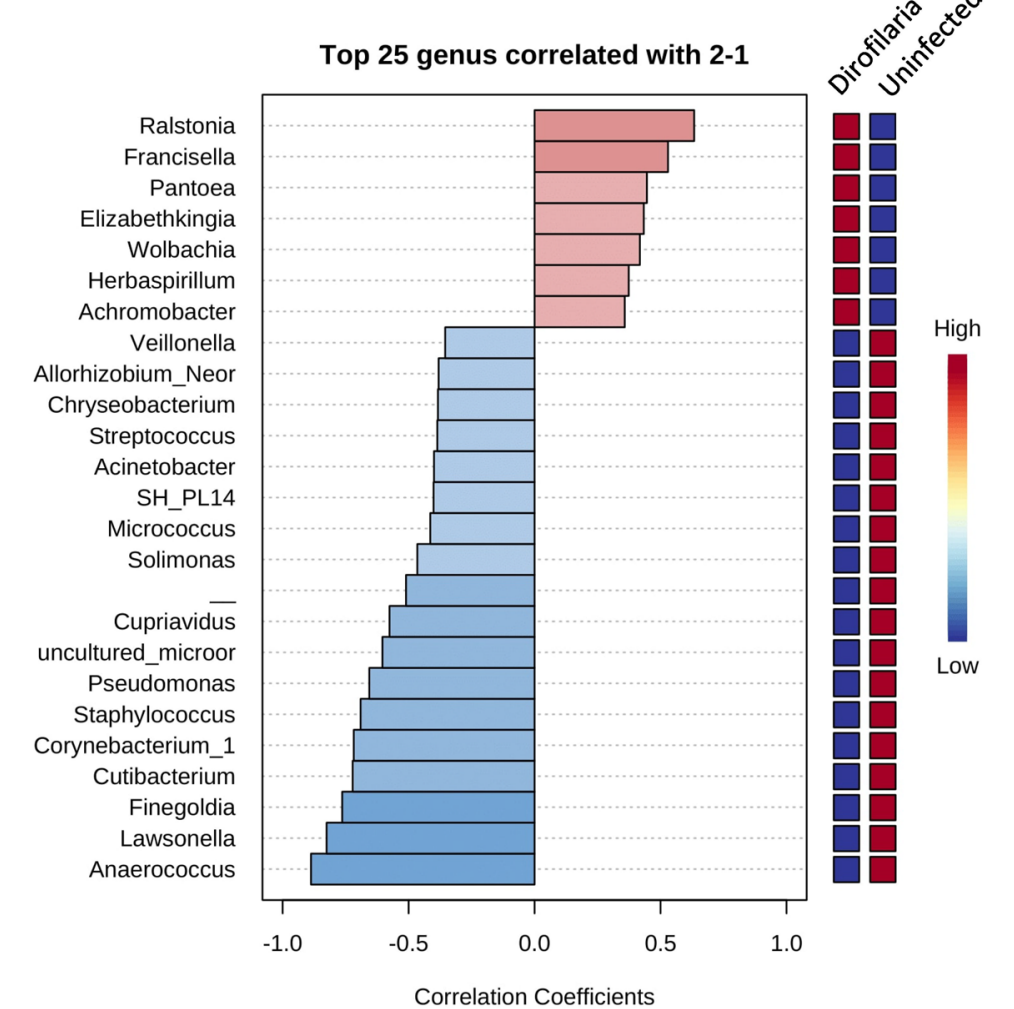

\section{b Actinobacteria}

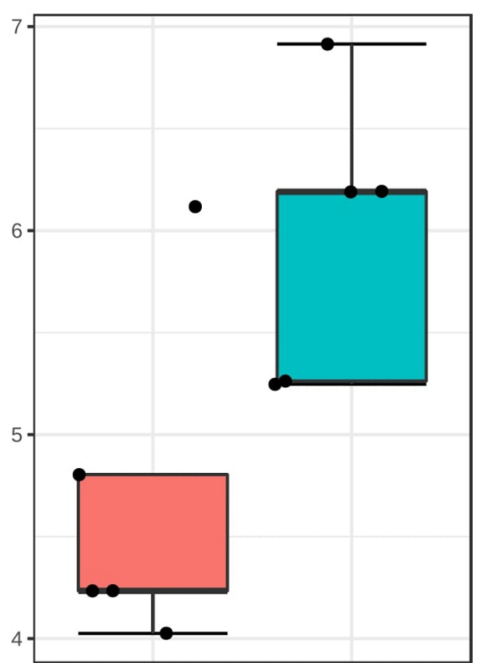

c Firmicutes

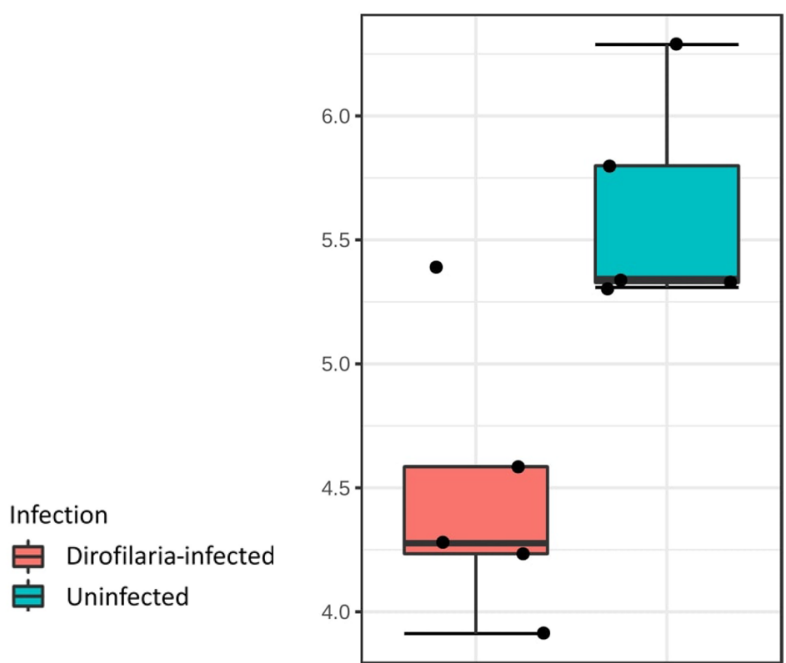

Fig. 6 Microbial community profile. Pattern correlation analysis and significant abundance analysis of top taxa identified in Dirofilaria immitis-infected and uninfected mosquitoes. SparCC correlation of top 25 genera showing bacteria with strong positive correlation with Dirofilaria immitis-infected mosquitoes (a), log-transformed count of bacteria of the phyla Actinobacteria (b) and Firmicutes (c) with significant differences in abundance between infected and uninfected mosquitoes (b FDR $=0.1440, d f=1, P=0.036 ; \mathbf{c} F D R=0.14401, d f=1, P=0.005$ ) 

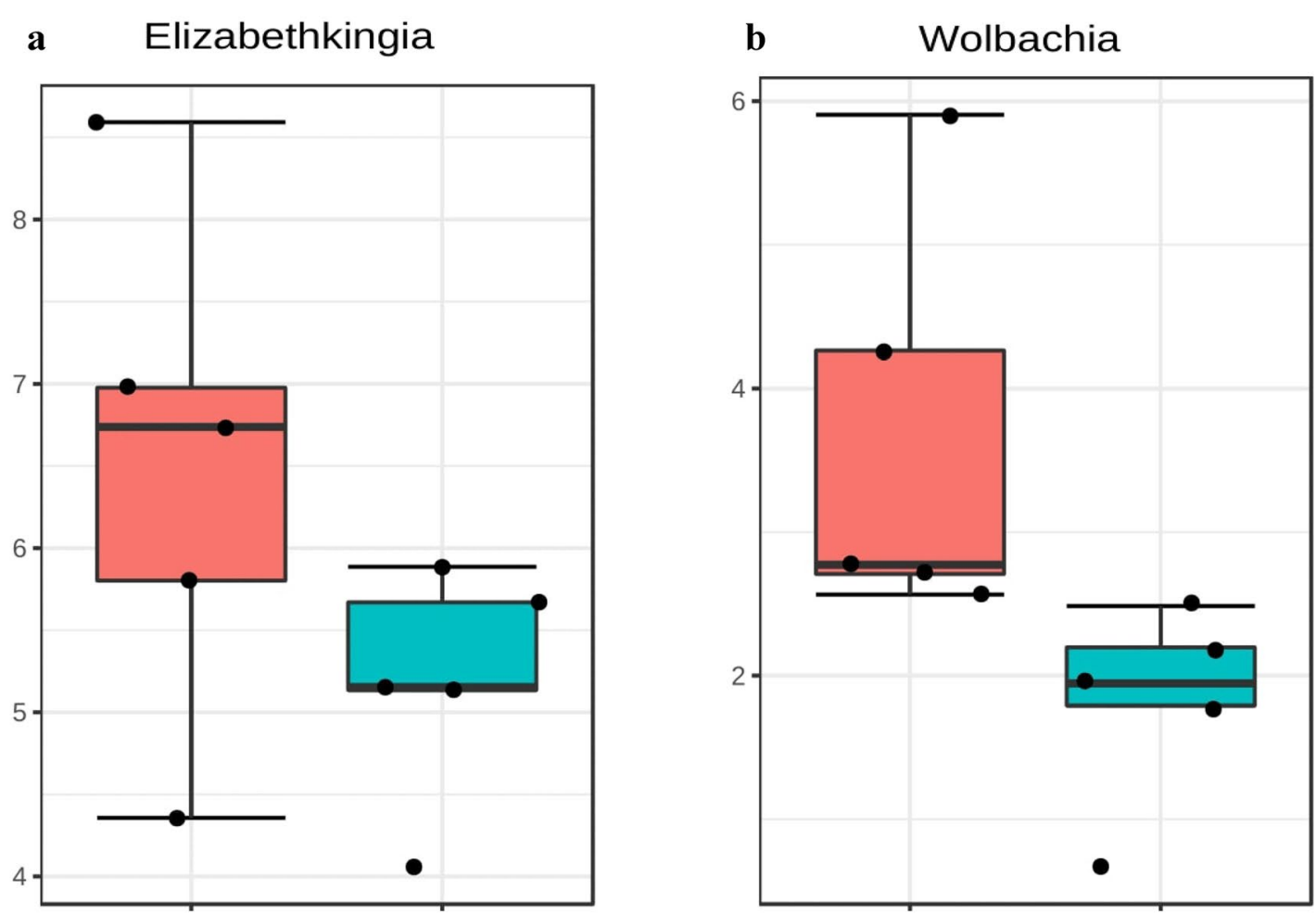

\section{c Pseudomonas}
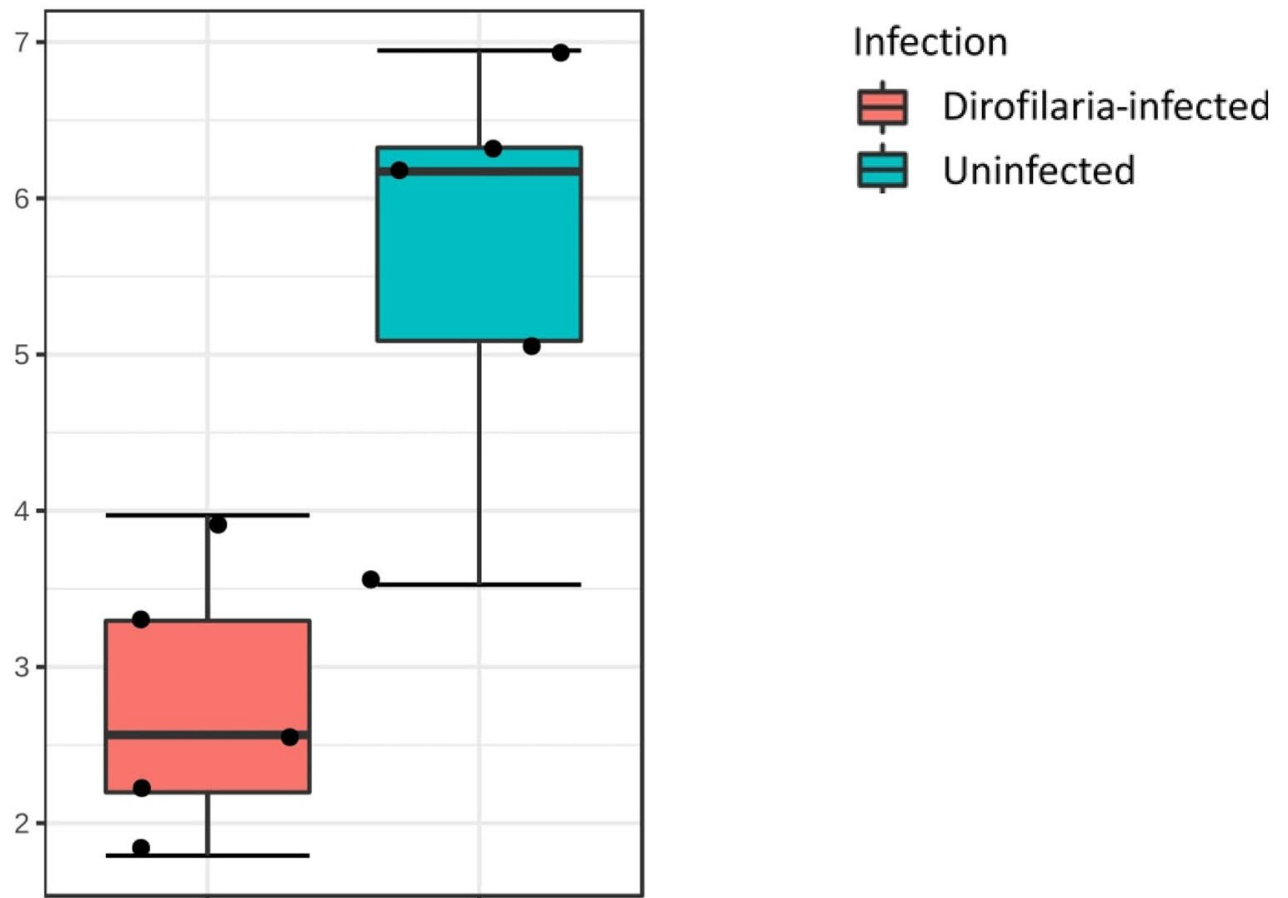

Fig. 7 Univariate analysis. Comparison for bacteria genera displaying significant differences in relative abundance between Dirofilaria immitis-infected and uninfected mosquitoes. Elizabethkingia ( $F D R=0.175, d f=1, P=0.015)$ and Wolbachia $(F D R=0.11448, d f=1, P=0.011)$ were significantly more abundant in infected compared to uninfected mosquitoes (a, b), while uninfected mosquitoes had more Pseudomonas $(F D R=0.12698, d f=1, P=0.015)(\mathbf{c})$ compared to infected mosquitoes 
of other mosquito species [46]. The role(s) D. immitis colonization and infection density plays in increasing the abundance of Enterobacter was beyond the scope of this study [47]; an elegant study by Cirimotich et al. [48] showed the inhibition of Plasmodium infection in Anopheles gambiae mosquitoes mediated by a bacterium designated as Enterobacter Esp_Z. The inhibition was due to the production of reactive oxygen species (ROS) by the bacteria. A pro-pathogen role was also recently associated with the genus Enterobacter, as it was shown that they produce Enhancins or Enhancins-like proteins, which facilitate pathogen colonization by degrading the peritrophic matrix [44]. If bacteria such as Enterobacter can also block $D$. immitis colonization by inducing ROS production will be interesting to see. Enterobacter hormaechei is another bacterium that demonstrated increased abundance in $D$. immitis infection as shown in Fig. 3. The genus Enterobacter have been proposed to aid in blood digestion in hematophagous insects due to their hemolytic activities [47]. Several reports have also identified different mosquito refractoriness or susceptibility to pathogen infection in the presence of different Enterobacter species. Cirimotich et al. [48] reported refractoriness of An. gambiae to Plasmodium infection in the presence of an Enterobacter species. Another species of Enterobacter (E. cloacae) was also reported to express a mucin-degrading Enhancin protein that breaks down the mucin component of the Ae. aegypti peritrophic matrix [44] although this was not shown to facilitate dengue virus infection.

Another bacteria genus that also increased with the presence of D. immitis infection is Elizabethkingia. Previous reports have identified this genus from the midgut of laboratory-reared [42] and field-collected [40] Ae. aegypti. These studies did not associate any known role to this bacterium. Bacteria in the genus Chryseobacterium, Ochrobactrum, Sphingobacterium and Pseudomonas were all present in higher abundance in the uninfected mosquito group. The detection of Chryseobacterium in our study agrees with similar detection in previous reports in laboratory-reared mosquitoes [49, 50]. Pseudomonas, a gram-negative Gammaproteobacteria was previously shown to have reduced abundance in Wolbachia-positive Ae. aegypti mosquitoes. A similar observation was also made in our study where the abundance of Pseudomonas was inversely correlated with the presence of $D$. immitis infection in the mosquito as shown in Fig. 2. Bahia et al. [51] reported blocking of the Plasmodium parasite by Pseudomonas putida isolated from An. gambiae.

Unexpectedly, only few bacteria species were differentially altered in D. immitis-infected and uninfected mosquitoes. Klebsiella oxytoca, a Gammaproteobacteria, was found at relatively similar abundance in infected and uninfected mosquitoes (Fig. 3). A study reported the detection of $K$. oxytoca from laboratory-reared and fieldcollected Cx. quinquefasciatus and Ae. aegypti [42]. The maintenance of this species by arthropods in both natural and artificial conditions could indicate an important role played by the bacteria. Another study reported that $K$. oxytoca reverse radiation induced loss of copulatory maintenance in male Ceratitis capitata [52]. A study on the fungicidal effects of bacterial colonies found on the domestic housefly eggs revealed that fungal growth is inhibited by the presence of $K$. oxytoca by producing antifungal metabolites and nutrient depletion [53]. Put together, we are proposing $K$. oxytoca as an important bacterium for Ae. aegypti with a likely endosymbiotic role, though further studies are still required to understand the specific roles of $K$. oxytoca in the mosquito biology including how it is maintained in the mosquito.

Another interesting observation from this study was the inverse correlation between $D$. immitis infection and microbial diversity and richness. In mosquitoes, the innate immune response is activated in the presence of invading pathogens as activation of the toll pathway and production of reactive oxygen species have been reported in mosquitoes challenged with Plasmodium [54] and filarial nematodes [55]. These immune effectors while countering pathogens, could in extension disrupt the normal mosquito microbiome community which could explain the overall reduction in the microbial richness observed from this study as shown in Fig. 4b, c. Our observation of reduced microbial richness in infected mosquitoes was in contrast to what was reported in a previous study which shows a more diverse microbiome in Plasmodium-infected Anopheles mosquitoes [56]. While our study did not factor the effect blood meal could have on the overall outcome of the microbial richness, few studies have reported reduction in the bacterial diversity following experimental feeding on host blood in Ae. aegypti [57] and An. gambiae [50].

\section{Conclusions}

This study fills a knowledge gap on the interaction between a mosquito vector and a filarial pathogen of veterinary significance. We were able to show that while some bacterial species were found to be present in both mosquito groups, the relative abundances of individual species changes with the infection status, with infected mosquitoes presenting a reduced microbial richness. This indicates a likely consequence of the nematode in altering favoring or inhibiting the growth of members of the bacterial community. Ongoing study focuses on the shift in the distribution of culturable bacteria taxa in infected and uninfected mosquitoes, while also comparing the 
effects of $D$. immitis colonization on the microbial diversity of different tissues of the Ae. aegypti mosquito.

\section{Supplementary information}

Supplementary information accompanies this paper at https://doi. org/10.1186/s13071-020-04218-8.

Additional file 1: Figure S1. Agarose gel electrophoresis of PCR amplified D. immitis 656-bp cox1 mtDNA gene confirming the infection status of Ae. aegypti. Figure S2. Quality plots of forward and reverse reads. Figure S3. Summary of sequence reads following demultiplexing. Figure S4. Relative abundance of bacteria phyla across individual mosquito samples analyzed. Figure S5. Relative abundance of bacteria families across individual mosquito samples analyzed. Figure S6. Relative abundance of bacteria genera across individual mosquito samples analyzed. Figure S7. Relative abundance of bacteria species across individual mosquito samples analyzed. Figure S8. Heat map analysis of the differential composition showing most abundant bacteria genera identified in this study. Figure $\mathbf{S 9}$. Phylogenetic reconstruction of the relative abundances of bacteria genera identified from both D. immitis-infected and uninfected mosquitoes.

\section{Acknowledgements}

The following reagents were provided by the NIH/NIAID Filariasis Research Reagent Resource Center through BEl Resources: Uninfected Canine Blood (Not Exposed to Filariae) NR-48922 and Dirofilaria immitis, Strain Missouri, Microfilariae in Dog Blood (Live) NR-4890. We also thank the University of Southern Mississippi's School of Biological, Environmental, and Earth Sciences Human Parasitology class participants (Fall 2019) for their technical assistance in extracting the genomic DNA and pathogen detection.

\section{Authors' contributions}

GD and SK conceived and designed the experiments. ASA, EN, AG, MCH, KW and SMN performed the experiments. ASA, GD and SK analyzed the data. GD and SK contributed reagents/materials/analysis tools. ASA, EN, AG, GD and SK wrote the paper. All authors read and approved the final manuscript.

\section{Funding}

This study was principally supported by a Pakistan-US Science and Technology Cooperation Program award (US Department of State), the National Institutes of General Medical Sciences \#P20 and GM103476 to the University of Southern Mississippi and the Department of Energy Office of Environmental Management under Award Number DE-EM0004391 to the University of Georgia Research Foundation. AG, MCH, KW and SMN were supported by the Eagle SPUR award from the USM Drapeau Center for Undergraduate Research.

\section{Availability of data and materials}

The datasets supporting the conclusion of this article are included within the article and its additional files. Raw data are available from the corresponding author upon request.

\section{Ethics approval and consent to participate}

Mosquitoes were blood-fed using an artificial membrane feeder. The protocol for the laboratory was approved by the Institutional Biosafety Committee.

\section{Consent for publication}

Not applicable.

\section{Competing interests}

The authors declare that they have no competing interests.

\section{Author details}

${ }^{1}$ Center for Molecular and Cellular Biosciences, School of Biological, Environmental, and Earth Sciences, The University of Southern Mississippi, Hattiesburg, MS 39406, USA. ${ }^{2}$ Savannah River Ecology Laboratory, University of Georgia, P.O. Drawer E, Aiken, SC 29802, USA. ${ }^{3}$ Warnell School of Forestry and Natural Resources, University of Georgia, Athens, GA 30602, USA.
Received: 17 February 2020 Accepted: 6 July 2020

Published online: 13 July 2020

\section{References}

1. Bowman DD, Atkins CE. Heartworm biology, treatment, and control. Vet Clin North Am Small Anim Pract. 2009;39:1127-58.

2. Spence Beaulieu MR, Reiskind MH. Comparative vector efficiency of two prevalent mosquito species for dog heartworm in North Carolina. J Med Entomol. 2020;57:608-14.

3. Theis JH. Public health aspects of dirofilariasis in the United States. Vet Parasitol. 2005;133:157-80.

4. Bowman DD, Liu Y, McMahan CS, Nordone SK, Yabsley MJ, Lund RB. Forecasting United States heartworm Dirofilaria immitis prevalence in dogs. Parasit Vectors. 2016;9:540.

5. Evans CC, Moorhead AR, Storey BE, Wolstenholme AJ, Kaplan RM. Development of an in vitro bioassay for measuring susceptibility to macrocyclic lactone anthelmintics in Dirofilaria immitis. Int J Parasitol Drugs Drug Resist. 2013;3:102-8.

6. McCall JW. The role of arthropods in the development of animal - models for filariasis research. J Georgia Ent Soc. 1981;16:283-93.

7. Morchón R, Carretón E, González-Miguel J, Mellado-Hernández I. Heartworm disease (Dirofilaria immitis) and their vectors in Europe-new distribution trends. Front Physiol. 2012;3:196.

8. Wang D, Bowman DD, Brown HE, Harrington LC, Kaufman PE, Mckay T, et al. Factors influencing U.S. canine heartworm (Dirofilaria immitis) prevalence. Parasit Vectors. 2014;7:264.

9. Ludlam KW, Jachowski LA Jr, Otto GF. Potential vectors of Dirofilaria immitis. J Am Vet Med Assoc. 1970;157:1354-9.

10. Drake J, Wiseman S. Increasing incidence of Dirofilaria immitis in dogs in USA with focus on the southeast region 2013-2016. Parasit Vectors. 2018;11:39.

11. Lok JB, Walker ED, Scoles GA. Filariasis. In: Eldridge BF, Edman JD, editors. Medical Entomology. Dordrecht: Kluwer Academic Publishers; 2000. p. 299-375

12. Ledesma N, Harrington L. Mosquito vectors of dog heartworm in the United States: vector status and factors influencing transmission efficiency. Top Companion Anim Med. 2011;26:178-85.

13. Guégan M, Zouache K, Démichel C, Minard G, Van VT, Potier P, et al. The mosquito holobiont: fresh insight into mosquito-microbiota interactions. Microbiome. 2018;6:49.

14. Engel P, Moran NA. The gut microbiota of insects - diversity in structure and function. FEMS Microbiol Rev. 2013;37:699-735.

15. Strand MR. The gut microbiota of mosquitoes: diversity and function. In: Wikel S, Aksoy S, Dimopoulos G, editors. Arthropod vector: controller of disease transmission, vol. 1. Cambridge, Massachusetts: Academic Press; 2017. p. 185-99.

16. Strand MR. Composition and functional roles of the gut microbiota in mosquitoes. Curr Opin Insect Sci. 2018;28:59-65.

17. Souza-Neto JA, Powell JR, Bonizzoni M. Aedes aegypti vector competence studies: a review. Infect Genet Evol. 2019;67:191-209.

18. Bonnet SI, Binetruy F, Hernández-Jarguín AM, Duron O. The tick microbiome: why non-pathogenic microorganisms matter in tick biology and pathogen transmission. Front Cell Infect Microbiol. 2017;7:236.

19. Alto BW, Lounibos LP, Mores CN, Reiskind MH. Larval competition alters susceptibility of adult Aedes mosquitoes to dengue infection. Proc Biol Sci. 2008;275:463-71.

20. Marchette NJ, Garcia R, Rudnick A. Isolation of Zika virus from Aedes aegypti mosquitoes in Malaysia. Am J Trop Med Hyg. 1969;18:411-5.

21. Martin E, Moutailler S, Madec Y, Failloux AB. Differential responses of the mosquito Aedes albopictus from the Indian Ocean region to two chikungunya isolates. BMC Ecol. 2010;10:8.

22. Erickson SM, Xi Z, Mayhew GF, Ramirex JL, Aliota MT, Christensen BM, et al. Mosquito infection responses to developing filarial worms. PLoS Negl Trop Dis. 2009;3:e529.

23. Ramirez JL, Souza-Neto J, Torres Cosme R, Rovira J, Ortiz A, Pascale JM, et al. Reciprocal tripartite interactions between the Aedes aegypti midgut microbiota, innate immune system and dengue virus influences vector competence. PLoS Negl Trop Dis. 2012;6:e1561. 
24. Dennison NJ, Jupatanakul N, Dimopoulos G. The mosquito microbiota influences vector competence for human pathogens. Curr Opin Insect Sci. 2014;3:6-13.

25. de la Fuente J, Antunes S, Bonnet S, Domingos AG, Estrada-Peña A, Johnson N, et al. Tick-pathogen interactions and vector competence: identification of molecular drivers for tick-borne diseases. Front Cell Infect Microbiol. 2017;7:114

26. Bourguinat C, Lee AC, Lizundia R, Blagburn BL, Liotta JL, Kraus MS, et al. Macrocyclic lactone resistance in Dirofilaria immitis: failure of heartworm preventives and investigation of genetic markers for resistance. Vet Parasitol. 2015;210:167-78.

27. Bourguinat C, Keller K, Bhan A, Peregrine A, Geary T, Prichard R. Macrocyclic lactone resistance in Dirofilaria immitis. Vet Parasitol. 2011;181:388-92.

28. Geary TG, Bourguinat C, Prichard RK. Evidence for macrocyclic lactone anthelmintic resistance in Dirofilaria immitis. Top Companion Anim Med. 2011;26:186-92.

29. Buxton BA, Mullen GR. Comparative susceptibility of 4 strains of Aedes aegypti (Diptera, Culicidae) to infection with Dirofilaria immitis. J Med Entomol. 1981;18:434-40.

30. Michalski ML, Griffiths KG, Williams SA, Kaplan RM, Moorhead AR. The $\mathrm{NIH-NIAID}$ filariasis research reagent resource center. PLoS Negl Trop Dis. 2011;5:e1261.

31. Dharmarajan $\mathrm{G}$, Walker KD, Lehmann T. Variation in tolerance to parasites affects vectorial capacity of natural Asian tiger mosquito populations. Curr Biol. 2019;29(3946-3952):e5.

32. Murata K, Yanai T, Agatsuma T, Uni S. Dirofilaria immitis infection of a snow leopard (Uncia uncia) in a Japanese zoo with mitochondrial DNA analysis. J Vet Med Sci. 2003;65:945-7.

33. Callahan BJ, McMurdie PJ, Rosen MJ, Han AW, Johnson AJ, Holmes SP. DADA2: High-resolution sample inference from Illumina amplicon data. Nat Methods. 2016:13:581-3.

34. Price MN, Dehal PS, Arkin AP. FastTree 2-approximately maximumlikelihood trees for large alignments. PLoS One. 2010;5:e9490.

35. Chong J, Liu P, Zhou G, Xia J. Using MicrobiomeAnalyst for comprehensive statistical, functional, and meta-analysis of microbiome data. Nat Protoc. 2020;15:799-821.

36. Dhariwal A, Chong J, Habib S, King IL, Agellon LB, Xia J. MicrobiomeAnalyst: a web-based tool for comprehensive statistical, visual and metaanalysis of microbiome data. Nucleic Acids Res. 2017;45(W1):W180-8.

37. Mancini MV, Damiani C, Accoti A, Tallarita M, Nunzi E, Cappeli A, et al. Estimating bacteria diversity in different organs of nine species of mosquito by next generation sequencing. BMC Microbiol. 2018;18:126.

38. Coon KL, Vogel KJ, Brown MR, Strand MR. Mosquitoes rely on their gut microbiota for development. Mol Ecol. 2014;23:2727-39.

39. Coatsworth H, Caicedo PA, Van Rossum T, Ocampo CB, Lowenberger C. The composition of midgut bacteria in Aedes aegypti (Diptera: Culicidae) that are naturally susceptible or refractory to dengue viruses. J Insect Sci. 2018;18:12.

40. Audsley MD, Seleznev A, Joubert DA, Woolfit M, O'Neill SL, McGraw EA. Wolbachia infection alters the relative abundance of resident bacteria in adult Aedes aegypti mosquitoes, but not larvae. Mol Ecol. 2018;27:297-309.

41. Degli Esposti M, Martinez Romero E. The functional microbiome of arthropods. PLoS One. 2017;12:e0176573.

42. Gazzoni Araújo Gonçalves G, Feitosa APS, Portela-Júnior NC, de Oliveira CMF, de Lima Filho JL, Brayner FA, et al. Use of MALDI-TOF MS to identify the culturable midgut microbiota of laboratory and wild mosquitoes. Acta Trop. 2019;200:105174
43. Yadav KK, Adhikari N, Khadka R, Pant AD, Shah B. Multidrug resistant Enterobacteriaceae and extended spectrum $\beta$-lactamase producing Escherichia coli: a cross-sectional study in National Kidney Center, Nepal. Antimicrob Resist Infect Control. 2015;4:42

44. Wu P, Sun P, Nie K, Zhu Y, Shi M, Xiao C, et al. A gut commensal bacterium promotes mosquito permissiveness to arboviruses. Cell Host Microbe. 2019;25(101-112):e5.

45. Coon KL, Brown MR, Strand MR. Gut bacteria differentially affect egg production in the anautogenous mosquito Aedes aegypti and facultatively autogenous mosquito Aedes atropalpus (Diptera: Culicidae). Parasit Vectors. 2016;9:375

46. Minard G, Mavingui P, Moro CV. Diversity and function of bacterial microbiota in the mosquito holobiont. Parasit Vectors. 2013;6:146.

47. Gaio Ade O, Gusmão DS, Santos AV, Berbert-Molina MA, Pimenta PF, Lemos FJ. Contribution of midgut bacteria to blood digestion and egg production in Aedes aegypti (L.) (Diptera: Culicidae). Parasit Vectors. 2011;4:105.

48. Cirimotich CM, Dong Y, Clayton AM, Sandiford SL, Souza-Neto JA, Mulenga $\mathrm{M}$, et al. Natural microbe-mediated refractoriness to Plasmodium infection in Anopheles gambiae. Science. 2011:332:855-8.

49. Chen S, Zhao J, Joshi D, Xi Z, Norman B, Walker ED. Persistent infection by Wolbachia wAlbB has no effect on composition of the gut microbiota in adult female Anopheles stephensi. Front Microbiol. 2016;7:1485.

50. Wang Y, Gilbreath TM 3rd, Kukutla P, Yan G, Xu J. Dynamic gut microbiome across life history of the malaria mosquito Anopheles gambiae in Kenya. PLoS One. 2011;6:e24767.

51. Bahia AC, Dong Y, Blumberg BJ, Mlambo G, Tripathi A, Ben-MarzoukHidalgo OJ, et al. Exploring Anopheles gut bacteria for Plasmodium blocking activity. Environ Microbiol. 2014;16:2980-94.

52. Ben Ami E, Yuval B, Jurkevitch E. Manipulation of the microbiota of massreared Mediterranean fruit flies Ceratitis capitata (Diptera: Tephritidae) improves sterile male sexual performance. ISME J. 2010;4:28-37.

53. Lam K, Thu K, Tsang M, Moore M, Gries G. Bacteria on housefly eggs, Musca domestica, suppress fungal growth in chicken manure through nutrient depletion or antifungal metabolites. Naturwissenschaften. 2009:96:1127-32

54. Molina-Cruz A, DeJong RJ, Charles B, Charles B, Gupta L, Kumar S, et al. Reactive oxygen species modulate Anopheles gambiae immunity against bacteria and Plasmodium. J Biol Chem. 2008;283:3217-23.

55. Edgerton EB, McCrea AR, Berry CT, Kwok JY, Thompson LK, Watson B, et al. Activation of mosquito immunity blocks the development of transmission-stage filarial nematodes. Proc Natl Acad Sci USA. 2020;117:3711-7.

56. Bassene H, Niang EHA, Fenollar F, Dipankar B, Doucouré S, Ali E, et al. 165 metagenomic comparison of Plasmodium falciparum-infected, and noninfected Anopheles gambiae and Anopheles funestus microbiota from Senegal. Am J Trop Med Hyg. 2018;99:1489-98.

57. Muturi EJ, Dunlap C, Ramirez JL, Rooney AP, Kim CH. Host blood-meal source has a strong impact on gut microbiota of Aedes aegypti. FEMS Microbiol Ecol. 2019:95:1093.

\section{Publisher's Note}

Springer Nature remains neutral with regard to jurisdictional claims in published maps and institutional affiliations.

Ready to submit your research? Choose BMC and benefit from:

- fast, convenient online submission

- thorough peer review by experienced researchers in your field

- rapid publication on acceptance

- support for research data, including large and complex data types

- gold Open Access which fosters wider collaboration and increased citations

- maximum visibility for your research: over 100M website views per year

At BMC, research is always in progress.

Learn more biomedcentral.com/submissions 